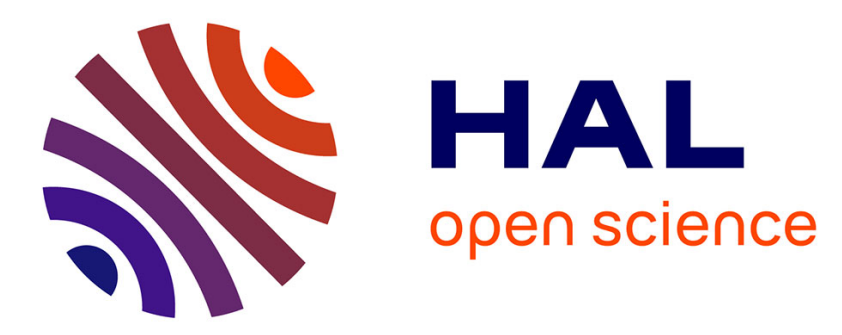

\title{
Universal pulses: A new concept for calibration-free parallel transmission
}

Vincent Gras, Alexandre Vignaud, Alexis Amadon, Denis Le Bihan, Nicolas Boulant

\section{- To cite this version:}

Vincent Gras, Alexandre Vignaud, Alexis Amadon, Denis Le Bihan, Nicolas Boulant. Universal pulses: A new concept for calibration-free parallel transmission. Magnetic Resonance in Medicine, 2017, 77 (2), pp.635-643. 10.1002/mrm.26148 . hal-02103499

\section{HAL Id: hal-02103499 https://hal.science/hal-02103499}

Submitted on 18 Apr 2019

HAL is a multi-disciplinary open access archive for the deposit and dissemination of scientific research documents, whether they are published or not. The documents may come from teaching and research institutions in France or abroad, or from public or private research centers.
L'archive ouverte pluridisciplinaire HAL, est destinée au dépôt et à la diffusion de documents scientifiques de niveau recherche, publiés ou non, émanant des établissements d'enseignement et de recherche français ou étrangers, des laboratoires publics ou privés. 


\title{
Universal pulses: a new concept for calibration-free parallel transmission
}

\author{
Authors and affiliations: \\ Vincent Gras, Alexandre Vignaud, Alexis Amadon, Denis Le Bihan, Nicolas \\ Boulant
}

CEA, DSV, I2BM, NeuroSpin, Unirs, Gif sur Yvette 91191, France

\section{Corresponding author:}

Nicolas Boulant, CEA, NeuroSpin, Gif sur Yvette, 91191 Cedex, France

e-mail: nicolas.boulant@ecea.fr

Tel: (+33)169087682 Fax: $(+33) 169087855$

Running head: Calibration-free parallel transmission with universal pulses

Word Count: 5244 


\begin{abstract}
Purpose: A calibration-free parallel transmission method is investigated to mitigate the radiofrequency $(\mathrm{RF})$ field inhomogeneity problem in brain imaging at $7 \mathrm{~T}$.

Methods: Six volunteers were scanned to build a representative database of RF and static field maps at 7T. Small-tip-angle and inversion pulses were designed with joint $\mathrm{k}_{\mathrm{T}}$-points trajectory optimization to work robustly on all six subjects. The returned "universal" pulses were then inserted in an MPRAGE sequence implemented on six additional volunteers without further field measurements and pulse optimizations. Similar acquisitions were performed in the circularly-polarized mode and with subject-based optimizations for comparison. Performance of the different approaches was evaluated by means of image analysis and computation of the flip angle normalized root mean square errors (NRMSE).
\end{abstract}

Results: For both the excitation and inversion, the universal pulses (NRMSE 11\%) outperformed the circularly-polarized (NRMSE 28\%) and RF shim modes (NRMSE 20\%) across all volunteers and returned slightly worse results than for subject-based optimized pulses (NRMSE 7\%).

Conclusion: RF pulses can be designed to robustly mitigate the RF field inhomogeneity problem over a population class. This appears as a first step towards another plug and play parallel transmission solution where the pulse design can be done offline and without measuring subject-specific field maps.

Keywords: Parallel transmission, ultra-high field, plug and play, RF pulse design 


\section{Introduction}

Ultra-high field (UHF) MRI has led to the development of new radiofrequency (RF) coils with parallel transmission (pTx) system architectures (1-5), offerring a better control of the RF field homogeneity, a crucial point at those field strengths to fully exploit the potential of these powerful scanners. Because hardware improvements alone have appeared not sufficient for that purpose, new compensating techniques were also developed at the level of the RF pulses, such as the "fast kz" or "spokes" method $(6,7)$, suitable for slice-selective excitation, and the " $\mathrm{k}_{\mathrm{T}}$-points" method for non-selective excitation (8). In many circumstances, these new RF pulses are promising alternatives to adiabatic pulses, whose applicability at UHF is often limited due to their long durations, high peak power and thus large SAR demands to achieve uniform excitation. Interestingly also, these methods, although applicable with standard single channel RF coils, benefit from the aforementioned hardware improvements, and reciprocally, the interest in using parallel transmission systems has grown with the development of these techniques. Today, a demonstration of the $\mathrm{k}_{\mathrm{T}}$-points parametrization and its potential with $\mathrm{pTx}$ exists for a variety of sequences used by radiologists (8-11). Unfortunately, it remains that for applying these methods, the transmit RF field (B1) and, in most cases, the static field $(\Delta \mathrm{B} 0)$ distributions of the subject must be known. To date, the measurement of those maps combined with data extraction, processing, and sophisticated pulse design can easily cumulate 15 minutes or more $(9,12)$, which decreases by the same amount the time available for acquiring clinically relevant data.

The need to measure B1 maps in pTx at each session comes from the dependence of the RF field distribution with the inserted load, an effect that usually cannot be neglected. Thus, at $\mathrm{UHF}$, the B1 distribution may change significantly if, for example, the position, size, dielectric constant or conductivity of the medium constituting the sample are changed (13). However, for a given type of examination - as far as we are concerned here, brain imaging in the head first supine position - and by careful positioning of the subject, these variables can change in practice only moderately. Moreover, at high field strength, the RF field distribution in the head is strongly influenced by the conductivity of the biological tissues (typically $\sim 0.67 \mathrm{~S} / \mathrm{m}$ for brain tissue at $300 \mathrm{MHz}$ ) which reduces penetration into the sample and hence prevents pure dielectric resonance $(13,14)$. As a consequence, the dependence of the field distribution in the sample with its shape is greatly softened. More recent works dedicated to this matter confirm the soundness of this idea (15). As another supporting argument, one can also refer to the RF safety 
concepts of parallel transmission reported by Graesslin et al. in (16), who propose to use electric field simulations on a representative set of human head models for local SAR prediction in real experiments. This approach hence relies on the reproducibility of the RF electric fields across subjects and suggests implicitly the same behavior for the RF magnetic field, given that the electric and magnetic fields are linked through Maxwell's equations. More quantitatively, it was found in (17) that the peak 10-g SAR for the same RF pulse could be amplified by $40 \%$ when considering 6 different head sizes and anatomies. Because the SAR varies quadratically with the amplitude of the three components of the electric field, again one can reasonably expect more moderate variations of the single component B1 field across different subjects. Based on this principle, pre-optimized RF shim solutions for different organs (18) and 2-spoke pulses robust over different breathing configurations (inhale and exhale) in the human heart (19) have already been designed.

In this work, we build on this observation to avoid systematic measurement of the RF and static field distributions for each subject, which are needed in most pulse design strategies. The design of non-selective pTx RF pulses qualified as universal is investigated: such pulses do not involve the subject-specific field distributions but yet are targeted to significantly improve performance compared to the standard circularly-polarized (CP) and RF shim modes. To ensure the "universality" of the pulses, we propose a design guided by a database of $\Delta \mathrm{B} 0$ and B1 maps acquired experimentally on a few representative subjects. Different RF pulse design approaches are then experimentally tested and compared by means of Magnetization Prepared Rapid Gradient Echo (MPRAGE) sequences acquired in vivo at 7T (20), whereby small tip angle and inversion pulses are investigated. The results are also compared quantitatively by computing simulated Flip Angle (FA) normalized root mean square errors (NRMSEs) while gray-white matter segmentation is performed to verify standard segmentation pipelines with the newly acquired data. Finally, the effect of $\Delta \mathrm{B} 0$ and $\mathrm{B} 1$ variability on the performance of the universal pulses is investigated by varying the size of the database and by analyzing the tolerance of the universal pulses to $\Delta \mathrm{B} 0$ mismatches.

\section{Theory}

With the goal of testing RF pulses in an MPRAGE sequence, small $\left(9^{\circ}\right)$ and large $\left(180^{\circ}\right)$ tip angle pulses were designed to achieve, in principle, optimal contrast between white matter 
$(\mathrm{WM})$ and gray matter $(\mathrm{GM})$ at $7 \mathrm{~T}$ when $\mathrm{TR}=2.6 \mathrm{~s}$ and $\mathrm{TI}=1.1 \mathrm{~s}$. The pulses in this case were non-selective so that we chose the $\mathrm{k}_{\mathrm{T}}$-points parametrization to tackle the problem. The FA homogenization problem with $\mathrm{N}_{\mathrm{kT}} \mathrm{k}_{\mathrm{T}}$-points on a pTx system equipped with $\mathrm{N}_{\mathrm{c}}$ transmitters consists in optimizing the RF complex coefficients and the locations in k-space of each $\mathrm{k}_{\mathrm{T}}$-point so as to minimize the deviation of the returned FA map from the desired nominal value. For one subject, this problem is often written as the following magnitude least-squares (MLS) problem (21):

$$
\begin{aligned}
& \min _{\mathbf{x}, \mathbf{k}}\left\|\mathrm{A}(\mathbf{x}, \mathbf{k})-\alpha_{\mathrm{t}}\right\|_{2}, \\
& (\mathbf{x}, \mathbf{k}) \in \mathbb{C}^{\mathrm{N}_{\mathrm{c}} \mathrm{N}_{\mathrm{kT}}} \times \mathbb{R}^{3 \mathrm{~N}_{\mathrm{kT}}},
\end{aligned}
$$

where $\mathbf{x}$ is the concatenation of the $\mathrm{N}_{\mathrm{c}} \times \mathrm{N}_{\mathrm{kT}}$ RF complex amplitudes, $\mathbf{k}$ is the concatenation of the $\mathrm{k}_{\mathrm{T}}$-points locations along each axis $\left(\mathrm{k}_{\mathrm{x}}, \mathrm{k}_{\mathrm{y}}\right.$ and $\left.\mathrm{k}_{\mathrm{z}}\right), \mathbf{A}$ is the Bloch operator that returns the FA generated by the corresponding RF and gradient waveforms for the $\mathrm{N}_{\mathrm{v}}$ voxels included in the brain mask of the subject of interest, and where $\alpha_{t}$ is the target FA (either $9^{\circ}$ or $180^{\circ}$ ). To generalize the concept of FA homogenization simultaneously over multiple subjects, we now seek the same optimal RF coefficients / trajectory pair $(\mathbf{x}, \mathbf{k})$ that minimize the maximum root mean square (RMS) error over $\mathrm{N}_{\mathrm{s}}$ subjects. For a set of $\mathrm{N}_{\mathrm{s}}$ different operators $\mathbf{A}_{1}, \cdots, \mathbf{A}_{\mathrm{Ns}}$, each one associated to subject-specific $\mathrm{B} 1$ and $\Delta \mathrm{B} 0$ distributions, the problem thus becomes:

$$
\begin{aligned}
& \min _{\mathbf{x}, \mathbf{k}}\left(\max _{1 \leq \mathrm{i} \leq \mathrm{N}_{\mathrm{s}}}\left\|\mathbf{A}_{\mathrm{i}}(\mathbf{x}, \mathbf{k})-\alpha_{\mathrm{t}}\right\|_{2}\right), \\
& (\mathbf{x}, \mathbf{k}) \in \mathbb{C}^{\mathrm{N}_{\mathrm{c}} \mathrm{N}_{\mathrm{kT}}} \times \mathbb{R}^{3 \mathrm{~N}_{\mathrm{kT}}} .
\end{aligned}
$$

As RF pulses must respect hardware and safety limits for the patient (22), we shall add to the latter problem a set of explicit constraints on the RF coefficients (variable $\mathbf{x}$ ). The constraints are usually fourfold $(23,24)$ : i) the global SAR constraint, written as $\mathrm{c}_{\mathrm{G}}(\mathbf{x}) \leq \mathrm{SAR}_{\mathrm{G}, \mathrm{max}}$; ii) the local SAR limit, which, with the use of the Virtual Observation Point (VOP) compression scheme $(25,26)$, is expressed as a set of $\mathrm{N}_{\mathrm{VOP}}(\simeq 200$ in this case $)$ conditions $\mathrm{c}_{10 \mathrm{~g}, \mathrm{~m}}(\mathbf{x}) \leq$ $\mathrm{SAR}_{10 \mathrm{~g} \text {,max }}, 1 \leq \mathrm{m} \leq \mathrm{N}_{\mathrm{VOP}}$; iii) the average power constraints for each transmit channel, written as $\mathrm{c}_{\mathrm{Pw}, \mathrm{n}}(\mathbf{x}) \leq \mathrm{P}_{\max } \quad\left(1 \leq \mathrm{n} \leq \mathrm{N}_{\mathrm{c}}\right)$; and iv) the peak power constraints, limiting in amplitude each RF sub-pulse for each transmit channel, and thus written as $\left|x_{j}\right| \leq V_{\max }, 1 \leq j \leq N_{c} \times N_{k T}, V_{\max }$ being the maximum RMS voltage available at every coil Tx-element input. The average power limit above maintains coil stability by preventing it from warming up too much. It is a forward power which, due to the presence of circulators in the power amplifiers, is independent of the 
load. Imperfect circulators can lead to deviations from this ideal behavior. But as shown in [12], these deviations are small with state of the art equipment and can be safely neglected. For SAR calculations, we made use of electromagnetic simulations on generic head models (16). Thus, the electric field maps generated by each coil element of the transmission array used throughout the study were computed with the HFSS finite element software (Ansys, Canonsburg, PA, USA) on two different head models (male and female) (27) and were exported on a $5 \mathrm{~mm}$ isotropic grid. The Q matrices (16) were first averaged over 10-g of contiguous tissue using a home-made region-growing algorithm and then compressed following the method of Lee et al. (26). Additional safety factors of $1.25,1.4$ and 1.55 were then successively applied to respectively take into account modeling errors (28), anatomic variability (17) and measurement uncertainties in the sequence monitoring hardware (29), thus yielding an overall safety factor of 2.7. The VOP matrices were provided to the scanner monitoring software which was then able to calculate in real-time an upper bound of the peak 10-g SAR based on the complex signals played by the power amplifiers and picked up by directional couplers. For completeness, the mathematical formulation used in this work for the design of universal RF pulses thus becomes the following:

$$
\begin{aligned}
\min _{\mathbf{x}, \mathbf{k}}\left(\max _{1 \leq \mathrm{i} \leq \mathrm{N}_{\mathrm{s}}}\left\|\mathbf{A}_{\mathrm{i}}(\mathbf{x}, \mathbf{k})-\alpha_{\mathrm{t}}\right\|_{2}\right), \\
(\mathbf{x}, \mathbf{k}) \in \mathbb{C}^{\mathrm{N}_{\mathrm{c}} \mathrm{N}_{\mathrm{kT}}} \times \mathbb{R}^{3 \mathrm{~N}_{\mathrm{kT}},} \\
\mathrm{c}_{\mathrm{G}}(\mathbf{x}) \leq \mathrm{SAR}_{\mathrm{G}, \text { max }} \\
\mathrm{c}_{10 \mathrm{~g}, \mathrm{~m}}(\mathbf{x}) \leq \mathrm{SAR}_{10 \mathrm{~g}, \text { max }}, 1 \leq \mathrm{m} \leq \mathrm{N}_{\mathrm{VOPs}}
\end{aligned}
$$

s. t.

$$
\begin{aligned}
& \mathrm{c}_{\mathrm{Pw}, \mathrm{n}}(\mathbf{x}) \leq \mathrm{P}_{\text {max }}, 1 \leq \mathrm{n} \leq \mathrm{N}_{\mathrm{c}} \\
& \left|\mathrm{x}_{\mathrm{j}}\right| \leq \mathrm{V}_{\text {max }}, 1 \leq \mathrm{j} \leq \mathrm{N}_{\mathrm{c}} \times \mathrm{N}_{\mathrm{kT}} .
\end{aligned}
$$

\section{Methods}

\section{Experimental set-up}

All measurements in this work were performed on a 7T Magnetom Scanner (Siemens Healthcare, Erlangen, Germany) equipped with an 8 channel transmit array (1 kW peak power per channel) and an AC84 head gradient system $(50 \mathrm{mT} / \mathrm{m}$ maximum amplitude and $333 \mathrm{~T} / \mathrm{m} / \mathrm{s}$ 
maximum slew rate). Our study was approved by a national ethic committee and subjects gave written informed consent. For both RF transmission and reception, a home-made transceiverarray head coil was used. The array consists of 8 stripline dipoles distributed every $42.5^{\circ}$ on a cylindrical surface of 27.6-cm diameter, leaving a small open space in front of the subject's eyes. All dipoles were tuned ideally at $297.18 \mathrm{MHz}$ corresponding to the proton Larmor frequency at $7 \mathrm{~T}$ and matched identically to a $50 \mathrm{Ohm}$ line impedance. Tuning and matching conditions were left unchanged throughout all measurements, including different volunteers. Up to second-order $\mathrm{B}_{0}$ shims were used prior to any imaging sequence.

\section{Database construction}

The $\mathrm{B} 1$ and $\Delta \mathrm{B} 0$ field distributions database was created from a population of six subjects ( 5 men, 1 woman, age: $25-28$ years, height: $1.65-1.80 \mathrm{~m}$, weight: $54-106 \mathrm{~kg}$ ). The static field offset was measured with a 3D multiple Gradient Recalled Echo (GRE) (2.5 mm isotropic resolution, matrix size $64 \times 96 \times 128, \mathrm{TR}=25 \mathrm{~ms}, \mathrm{TE}=5 / 6.5 / 8 \mathrm{~ms}, \mathrm{TA}=3 \mathrm{~min}$ ) while the reconstruction of the 8 complex transmit B1 fields in 3D was based on a multi-slice interferometric XFL acquisition (30-32) (5 mm isotropic resolution, matrix size $40 \times 64 \times 40, \mathrm{TR}=20 \mathrm{~s}, \mathrm{TA}=4$ min $40 \mathrm{~s}$ ). The 3D GRE data was also exploited for brain mask extraction via the FSL BET program (33) to isolate the voxels of interest, which were then used in the optimizations. The data analysis of the $n^{\text {th }}$ subject $\left(1 \leq \mathrm{n} \leq \mathrm{N}_{\mathrm{s}}=6\right)$, resulted in the creation of $\left.\mathrm{i}\right)$ a collection of positions $\mathbf{R}(\mathrm{n}) \in \mathbb{R}^{\mathrm{N}_{\mathrm{v}, \mathrm{n}} \times 3}$ (each row corresponding to a given set of $\mathrm{x}, \mathrm{y}$ and z-positions), ii) the measured transmit field distributions for each voxel in the brain and for each channel $\mathbf{B}_{1}(\mathrm{n}) \in \mathbb{C}^{\mathrm{N}_{\mathrm{v}, \mathrm{n}} \times \mathrm{N}_{\mathrm{c}}}$ and iii) the measured static field offset for each voxel $\Delta \mathbf{B}_{\mathbf{0}}(\mathrm{n}) \in \mathbb{R}^{\mathrm{N}_{\mathrm{v}, \mathrm{n}} \times 1}$. The vertical concatenation of those $\mathrm{N}_{\mathrm{s}}=6$ measurements then formed the position matrix $\mathbf{R}$, the transmit RF field matrix $\mathbf{B}_{1}$ and the static field offset vector $\Delta \mathbf{B}_{\mathbf{0}}$ of our so-called field database.

\section{Universal RF pulse design}

Using Eq. 3 and the field database described above, two RF pulses were generated with the $\mathrm{k}_{\mathrm{T}}$ point method: an RF pulse with a nominal FA of $9^{\circ}$, referred as the excitation pulse, and an RF pulse with a nominal FA of $180^{\circ}$ (inversion pulse). The excitation and inversion pulses were parametrized with 5 and $7 \mathrm{k}_{\mathrm{T}}$-points of total durations 0.7 and $4 \mathrm{~ms}$ respectively. Gradient blip durations were $60 \mu \mathrm{s}$ in all cases. In the MPRAGE sequence, both the small flip angle and inversion pulses contribute to the 10-s and 6-min time-averaged SAR and average power. 
Hence, to ensure compliance with the IEC guidelines (22) and for simplicity, the constraints for the train of excitation pulses $(=160)$ and inversion pulse were defined as $\mathrm{SAR}_{\mathrm{G}}, \mathrm{max} /$ $\mathrm{SAR}_{10 \mathrm{~g}, \max } / \mathrm{P}_{\max }=1.6 \mathrm{~W} / \mathrm{kg} / 6 \mathrm{~W} / \mathrm{kg} / 4 \mathrm{~W}$ and $1.6 \mathrm{~W} / \mathrm{kg} / 4 \mathrm{~W} / \mathrm{kg} / 4 \mathrm{~W}$ respectively (23), while the two pulses were optimized independently. Those limits take into account both the flip angle values (small versus large) and the duty cycles (large versus small) of the pulses used in the sequence, and appeared as nearly optimal for the implementation of the MPRAGE sequence used in this work. The maximum RMS voltage at the coil input constraint, $\mathrm{V}_{\max }$ in Eq. 3, was set to 150 Volts. In both cases, the RF coefficients were first initialized by using the variable exchange (VE) method (21) with the initial phase configuration returned by the CP-mode and an initial star-shaped k-space trajectory (9). A large Tikhonov regularization factor was added to make sure that the returned solutions initially satisfied all constraints, regardless of pulse performance. Starting from the solution returned by the VE method, the RF coefficients and the blipped k-space trajectory were then optimized simultaneously, under explicit SAR and hardware constraints, using the active-set (A-S) algorithm of Matlab (The Mathworks, Natick, MA, USA), chosen for this optimization problem for its speed and robustness $(23,34)$. Convergence was assumed if the change of the optimized variables and the first derivative of the Lagrangian reached a value below $10^{-6}$ and $10^{-5}$ respectively. A voxel-by-voxel Bloch integration was performed for both optimizations (excitation and inversion pulses) so that no small tip angle approximation was used in our implementation. To speed up the calculation, the objective function value and its gradient were computed using finite differences on a GPU device (Nvidia, Santa Clara, CA, Tesla K20c) with single (32 bit) precision combined with a Xeon E5-2670 bi-processor and 128 GB of RAM. The derivatives of the constraints made use of analytical formulae detailed in (23). With the 6-subject field database considered here and composed of about 72000 voxels, one evaluation of the objective function and its gradient took less than $300 \mathrm{~ms}$ for the inversion pulse.

\section{Validation protocol}

After running the 3D GRE and XFL sequences for the characterization of the $\triangle \mathrm{B} 0$ and $\mathrm{B} 1$ distributions on six different new subjects (4 men, 2 women, age: 23-27 years, height: 1.69$1.80 \mathrm{~m}$, weight: $54-82 \mathrm{~kg}$ ), i.e. not included in the field database, three MPRAGE acquisitions differing in the respective designs of the excitation and the inversion pulses were performed on them. One acquisition was implemented with an adiabatic hyperbolic secant pulse (35) of 10 ms duration and at maximum power allowed by the scanner for the inversion, and a rectangular 
pulse for excitation, both played in a CP-mode configuration. Another acquisition was performed with the universal pulses designed offline and based on our previously acquired field database. Finally, for the last acquisition, the universal pulses were replaced by $\mathrm{k}_{\mathrm{T}}$-point shapes tailored RF pulses, obtained more classically by minimizing the objective function given by Eq. 1, i.e. with the subject-specific field maps and same constraints and parameters as for the universal RF pulses. Throughout, the sequence parameters were TI $=1100 \mathrm{~ms}$, TR $=2600 \mathrm{~ms}$, $\mathrm{TE}=3 \mathrm{~ms}$, nominal $\mathrm{FA}=9 \circ$, readout bandwidth $=240 \mathrm{~Hz}$, echo train length $(\mathrm{ETL})=160,1 \times$ $1 \times 1 \mathrm{~mm}^{3}$ resolution with a $160 \times 240 \times 256$ matrix in sagittal acquisition, and TA $=9$ min . Knowledge of the subject-specific $\mathrm{B} 1$ and $\Delta \mathrm{B} 0$ maps allowed subsequent computation of the RF pulse performances for the universal, adiabatic inversion and square excitation pulses. For the latter two pulses, both the CP and RF-shim modes were evaluated, the RF-shim configuration being designed for each subject still under explicit hardware and SAR constraints and including both amplitude and phase optimization with the A-S technique (Eq. 1, $\mathrm{N}_{\mathrm{kT}}=1$ ). That way, the theoretical performance of the universal pulses could be quantified and compared to more standard subject-based optimizations. Finally, SPM12 (36) was used to perform WMGM segmentation after bias field correction to analyze the separability of the WM-GM signal intensities in the different acquisitions and to verify standard segmentation pipelines with the newly acquired data.

\section{Database size effect}

The measurements of the validation protocol were performed with universal pulses designed with six $B 1$ and $\Delta B 0$ maps (those of subjects $\# 1$ to \#6). The acquisitions performed on six additional subjects (subjects \#7 to \#12) provided additional B1 and $\Delta \mathrm{B} 0$ maps. To analyze the impact of the size of the database, we thus investigated numerically the following two extreme scenarios: i) a database composed of 1 subject only and ii) a database composed of all but 1 subject. After computing in both cases the respective universal pulses, the NRMSEs returned by the pulses were then evaluated by means of Bloch simulations across the so-called test subjects, i.e. those which were not included in the investigated database.

\section{Tolerance to static field deviations}

The robustness of the universal pulses with respect to $\Delta \mathrm{B} 0$ deviations inherent to the intersubject $\triangle \mathrm{B} 0$ variability was analyzed as follows: for each subject a Bloch simulation was performed with the universal pulse as input to calculate the NRMSE of the FA distribution in 
the brain after applying constant $\Delta \mathrm{B} 0$ offsets, ranging from -200 to $200 \mathrm{~Hz}$, to the initially measured $\Delta \mathrm{B} 0$ maps, and for both the excitation and inversion pulses. The same calculation was then performed by replacing the universal pulse with the subject-based tailored pulses. The two NRMSE deviations were then compared to establish whether the universal pulses are more robust with respect to static field deviations.

\section{Results}

Figure 1 reports the measured axial B1 maps at isocenter, in amplitude and phase, for the 12 subjects and for the 8 transmit channels. Qualitatively, the maps reveal a high degree of similarity across subjects. The subject-to-subject B1 correlations are displayed in Fig. 2 in the form of eight $12 \times 12$ correlation matrices ( 1 matrix per channel). The lowest magnitude of the complex correlation coefficients was 0.82 and corresponded to the first channel (TX\#1) and was due to an atypical position of subject \#10 in the anterior to posterior direction due to pads requested for comfort. On average, the average correlation between B1 maps was $\sim 0.95 \pm 0.02$ throughout channels. The correlation of the $\Delta \mathrm{B} 0$ maps across subjects on the other hand was poor, with values ranging from -0.13 to 0.85 . For each transmission channel, the mean B1 magnitude over the brain varied at most by $\pm 10 \%$ across subjects, except for subject \#10, again, where $20 \%$ variation was observed on channels 4 and 5 (located at the back of the head).

The NRMSEs for the excitation and inversion pulses as well as the corresponding FA histograms across all subjects are reported in Fig. 3.a-b and Fig. 3.c-d respectively. All numbers reported in Fig. 3 were computed through the use of the subject-specific field maps and Bloch

equation simulations. While the design of the subject-based RF-shim and $\mathrm{k}_{\mathrm{T}}$-points tailored pulses naturally made use of those maps, the design of the universal pulses on the other hand made use of the field maps only of subjects \#1-6 constituting the field database. In Fig. 3, a striking observation is that the universal pulses performed significantly better than the $\mathrm{CP}$ and the subject-based RF shim configurations throughout, even for the subjects not contained in the database (subjects \#7-12). In some cases even, e.g. subject \#7, the universal pulses performed even better than for some subjects contained in the database. For the inversion pulse, sometimes the histogram of the RF-shim scenario in Fig. 3.d appeared more peaked than for the universal pulse, but it was at the detriment of a longer FA distribution tail which deteriorated the NRMSE. For both the inversion and small tip angle pulses, the worst-case NRMSE for the universal pulses was $13 \%$ (subject \#8), which is typically the inhomogeneity obtained at $3 \mathrm{~T}$ on a human 
brain and in a CP mode (37), while the average NRMSE reached around $11 \%$. Not surprisingly, the subject-based $\mathrm{k}_{\mathrm{T}}$-point tailored pulses performed better than the universal pulses (NRMSE 7 \%). The gain, however, appears relatively marginal.

The MPRAGE images for subjects \#7-12 (i.e. not contained in the field database) are provided in Fig. 4 for the CP, universal and subject-based tailored pulses (left to right) whereby no correction of the coil reception profile was performed. Compared to the CP-mode pulses, the use of the universal pulses clearly restored the contrast between gray and white matter, notably in the cerebellum and temporal lobes. In general the tailored pulses enabled a better mitigation of the susceptibility artefacts.

Figure 5 reports the signal intensity histograms over the pooled white and gray matter masks for subjects \#7-12, after bias field correction and segmentation performed by SPM12 (36). The general trend that can be observed is the reappearance of the GM pool when switching from the CP mode to the universal pulses. For subjects \#7, \#9 and \#10, the histograms obtained with the universal pulses and with subject-based tailored pulses are in fact similar.

The analysis of the impact of the database size on pulse performance led to the following results: including only one subject in the database (subject \#1) clearly showed no robustness with such a poor population sample. In this case, the FA NRMSEs for the 11 test subjects ranged from $15.9 \%$ (subject \#3) to $30.5 \%$ (subject \#6) for the inversion pulse while the NRMSE for subject \#1 was $8.2 \%$ (corresponding to the performance of the subject-based tailored pulse). Including 11 subjects (subjects \#1 to \#11) in the database returned more uniform NRMSEs across database subjects $(\mathrm{NRMSE}=11.1 \pm 0.4 \%$ ) but closer to the minimized worst-case scenario $(11.4 \%)$, while the NRMSE evaluation on the unique test subject (subject \#12) returned $10.4 \%$.

The deviation of the NRMSE versus an additional $\triangle \mathrm{B} 0$ offset is reported in Fig. 6.a and Fig. 6.b for both the excitation and inversion pulses. The simulation results are provided for subjects \#7-12 and are shown in solid and dashed lines for the universal pulses and subjectbased tailored RF pulses respectively. The standard deviation of the measured $\Delta \mathrm{B} 0$ maps, displayed in Fig. 6.c, reveals a larger variability of $\Delta \mathrm{B} 0$ in the vicinity of air cavities (standard deviation $\sim 50 \mathrm{~Hz}$ ), which can impact negatively the performance of universal pulses in those regions. Yet, as shown in Fig. 6.a and Fig. 6.b, from the perspective of the NRMSE, one can 
see that the returned solutions for the universal pulses are more robust than for standard subjectbased optimizations with respect to $\Delta \mathrm{B} 0$ deviations.

\section{Discussion}

We have reported in this work an approach to design RF pulses that are robust with respect to head size, anatomy and position variations, and which thus require neither subjectspecific field measurements nor online pulse design. A somewhat similar concept is described in (18) where different RF-shim configurations were pre-calculated over different numerical models. Generalizing the concept to more complex waveforms, e.g. with $\mathrm{k}_{\mathrm{T}}$-points, was not trivial given that the $\mathrm{k}_{\mathrm{T}}$-points parametrization employs gradient blips which affect the voxels differently depending on their positions. For the same subject, but with different B1/ $\mathrm{B} 0$ map configurations on the heart during the breathing cycle (inhale and exhale), the same idea was also successfully employed in (19) with spokes. Spoke-pulses being in general more narrowband, the task of homogenizing the excitation simultaneously over several subjects may be more challenging and it remains to be established and verified experimentally to what extent the same concept can be applied successfully in 2D applications. On a positive note however, the work reported in (38) showed that the optimal k-space trajectory for 2-spoke pulses seemed robust across subjects and slice placement.

The universal pulses rely on a representative field database pre-acquired and for which the design is performed offline and under explicit hardware and SAR constraints. They are thus valid for a given field strength, body part, patient position (here, head first supine) and essentially also for a given pTx RF coil. Assuming a certain degree of similarity in the B1 and $\Delta \mathrm{B} 0$ maps among the tested population, the pulses then could be blindly and successfully applied on new subjects without subject-specific field map measurements and pulse calculations. With the tools and methods developed in the laboratory, for an MPRAGE sequence, this currently saves about 15 minutes (field measurements, data analysis, data transfer, brain mask extraction and pulse design including both RF and k-space trajectory optimization under constraints for a small and a large tip angle pulse). Importantly, the work shows that the development and application of universal pulses could make the use of the underlying parallel transmission hardware completely transparent to the user, as in (39), since no pTx expertise would be necessarily required to run the MR exam. The design of the universal 
pulses could be performed offline once and for all by MR experts and could be stored in corresponding pTx pulse libraries. Without time constraints, computation time also would become less problematic so that more thorough optimization approaches could be tested with, for example, numerous initializations of the non-convex optimization problem given in Eq. 3 to possibly obtain a solution close to the global optimum (34). Moreover, within this new framework, lots of different configurations could still be conceived, making the use of universal pulses flexible and powerful. As an example, a pulse portfolio with the same flip angles but different SAR levels (with SAR look ahead tables) could be created to accommodate different duty-cycles and for various MR sequences.

The field database used to design the universal pulses here contained 6 subjects. Six additional and different subjects were recruited to test their performance in an MPRAGE sequence. The universal pulses systematically outperformed the CP and RF-shim modes for both the excitation and inversion pulses (even with adiabatic modulations) (Fig. 3 and 4), with a substantial improvement in NRMSE. In this sense, the test of universal RF pulses on the 6 additional subjects never failed. Experimentally, as shown in Fig. 4 and 5, it was found that the universal pulses could achieve good inversion and excitations to restore the contrast between gray and white matter mainly in the cerebellum, temporal, occipital and frontal lobes. Naturally, the performance of the proposed pulses is lower than for subject-based tailored pulses. In this study however, the worst-case NRMSE obtained with a universal pulse at $7 \mathrm{~T}$ matched the performance of a CP configuration at 3T (Fig. 3. a-b) (37). Having a field database made of several subjects to guide the RF pulse design also was found to be essential to achieve robustness across volunteers. The calculations indicated that increasing the size of the database also likely helps achieving greater robustness. On the other hand, a very atypical and unlikely scenario could then penalize the rest. The use of other performance metrics like the mean NMRSE over all subjects in the database instead of the worst-case NRMSE employed throughout this study could possibly attenuate this effect.

Future work will consist in improving the performance of the universal pulses further. As shown in (34), a higher number of $\mathrm{k}_{\mathrm{T}}$-points as well as more initial k-space trajectory candidates should lead to smaller NRMSEs. The duration of the sub-pulses in the $\mathrm{k}_{\mathrm{T}}$-points could also be a valuable degree of freedom to optimize in order to be intrinsically more robust with respect to large $\Delta \mathrm{B} 0$ excursions. All these optimization considerations add additional computation complexity and time requirements. Nevertheless, being done offline, this can be more easily afforded. In our current design of universal pulses, the peak amplitude and 10-g 
SAR constraints were saturated so that additional improvements could also be obtained with more efficient coils or by relaxing the safety margins used in the SAR calculations. In the latter case, this could be achieved for instance by improving the fidelity of the numerical electromagnetic simulations (28), or if less conservative measures could be made regarding the uncertainties in the monitoring hardware (29). For the same purpose, other approaches based on temperature-regulated RF pulse design could be attempted as well (40), whereby the use of universal pulses within that context would considerably simplify exam planning since all optimizations and temperature calculations could be performed, again, offline.

More work is required to analyze the reproducibility of the field maps in order to maximize it. Certain variability in the $\Delta \mathrm{B} 0$ maps across subjects was indeed observed with correlations ranging from -0.13 to 0.85 and could presumably be improved by always starting from a more appropriate or perhaps the same set of shim currents. The problem as posed in Eq. 3 incorporates the $\Delta \mathrm{B} 0$ maps of different subjects but does not include additional $\Delta \mathrm{B} 0$ robustness constraints in the pulse design. By considering several subjects, the results in Fig. 6 simply revealed that the returned universal pulses were simply more broadband from the NRMSE point of view than standard subject-based optimizations to counteract the subject-tosubject variations in $\Delta \mathrm{B} 0$.

In this study, no particular screening in the procedure for the recruitment of the volunteers was employed, although the age was, involuntarily, quite uniform. Gender in the database also was not very representative of the general population. This naturally points to the non-optimality of our database for the same population and that efforts should be invested to carefully characterize the key criteria to sample in a given field database. For optimality purposes, one can easily imagine different universal pulses optimized for different classes of population, categorized by some criteria that remain to be identified (head circumference, oval or round head, etc). For head geometry as key criterion, fitting the head shape to a threedimensional ellipsoid in order to match it to the closest database in the atlas seems a viable approach (41). It remains also to be studied experimentally or perhaps through the use of numerical simulations which types of variations one should try to minimize for a given class of population. Some results presented here suggest that position is more critical than head size. But again, quite surprisingly, this noticeable difference in the field maps for subject \#10 did not engender a very different performance for the universal pulses (Figs. 3-5). However in general, 
the consequences of incorrect database matching, besides perhaps the risk to return non-optimal pulses, for the moment are unknown to the authors and remain to be studied.

Last, the applicability of universal pulses on other organs such as the abdomen clearly would be more challenging given the more drastic variability in sizes and the more severe variations of RF transmission profiles. But again, performance could be regained by matching a particular subject to the appropriate field database. Finally, on a positive note, one important milestone would be to simply return universal pulses with substantially more performance than with RF-shim solutions, i.e. an achievement that many could benefit from and which, according to the results reported here, appears more reachable. The remaining non-optimality of the proposed solutions would then be the price to pay for this plug and play implementation.

\section{Conclusion}

A method based on universal pulses to mitigate the RF field inhomogeneity problem at UHF, without subject-specific $\mathrm{B} 1$ and $\Delta \mathrm{B} 0$ map measurements, was presented. The proof of concept was validated with numerical simulations and measurements performed in vivo with nonselective 3D brain imaging at $7 \mathrm{~T}$. The results are very encouraging as they suggest that the use of parallel transmission could become completely transparent to the user, thus simplifying considerably the workflow and offering another plug-and-play pTx solution (39) to make the great potential of UHF scanners more accessible to anyone.

\section{Acknowledgements}

The authors wish to thank Dr. Lucie Hertz-Pannier, Dr. Pierre Brugières, Dr. Franck Mauconduit and Dr. Guillaume Ferrand for valuable discussions. The research leading to these results has received funding from the European Research Council under the European Union's Seventh Framework Program (FP7/2013-2018) / ERC Grant Agreement n. 309674. 


\section{References}

[1] Katscher U, Börnert P, Leussler C, van den Brink JS. Transmit SENSE. Magn Reson Med 2003;49:144-150.

[2] Adriany G, Van de Moortele PF, Wiesinger F, Moeller S, Strupp JP, Andersen P, Snyder C, Zhang X, Chen W, Pruessmann KP, Boesiger P, Vaughan T, Ugurbil K. Transmit and receive transmission line arrays for 7 Tesla parallel imaging. Magn Reson Med 2005;53:434-445.

[3] Ullmann P, Junge S, Wick M, Seifert F, Ruhm W, Hennig J. Experimental analysis of parallel excitation using dedicated coil setups and simultaneous RF transmission on multiple channels. Magn Reson Med 2005;54:994-1001.

[4] Setsompop K, Wald LL, Alagappan V, Gagoski B, Hebrank F, Fontius U, Schmitt F, Adalsteinsson E. Parallel RF transmission with eight channels at 3 Tesla. Magn Reson Med 2006;56:1163-1171.

[5] Grissom W, Yip Cy, Zhang Z, Stenger VA, Fessler JA, Noll DC. Spatial domain method for the design of RF pulses in multicoil parallel excitation. Magn Reson Med 2006;56:620-629.

[6] Saekho S, Boada FE, Noll DC, Stenger VA. Small tip angle three-dimensional tailored radiofrequency slab-select pulse for reduced B1 inhomogeneity at $3 \mathrm{~T}$. Magn Reson Med, 2005;53:479-484.

[7] Saekho S, Yip Cy, Noll DC, Boada FE, Stenger VA. Fast-kz three-dimensional tailored radiofrequency pulse for reduced B1 inhomogeneity. Magn Reson Med 2006;55:719-724.

[8] Cloos MA, Boulant N, Luong M, Ferrand G, Giacomini E, Le Bihan D, Amadon A. kTpoints: Short three-dimensional tailored RF pulses for flip-angle homogenization over an extended volume. Magn Reson Med 2012;67:72-80.

[9] Cloos MA, Boulant N, Luong M, Ferrand G, Giacomini E, Hang MF, Wiggins CJ, Bihan DL, Amadon A. Parallel-transmission-enabled magnetization-prepared rapid gradient-echo T1weighted imaging of the human brain at 7T. NeuroImage 2012;62:2140-2150.

[10] Massire A, Vignaud A, Robert B, Le Bihan D, Boulant N, Amadon A. Paralleltransmission-enabled three-dimensional T2-weighted imaging of the human brain at 7 Tesla. Magn Reson Med 2014;73:2195-2203. 
[11] Eggenschwiler F, O’Brien KR, Gruetter R, Marques JP. Improving T2-weighted imaging at high field through the use of kT-points. Magn Reson Med 2014;71:1478-1488.

[12] Deniz CM, Alon L, Brown R, Zhu Y. Subject- and resource-specific monitoring and proactive management of parallel radiofrequency transmission. Magn Reson Med 2015. doi: $10.1002 / \mathrm{mrm} .25828$.

[13] Yang QX, Wang J, Zhang X, Collins CM, Smith MB, Liu H, Zhu XH, Vaughan JT, Ugurbil K, Chen W. Analysis of wave behavior in lossy dielectric samples at high field. Magn Reson Med 2002;47:982-989.

[14] Van de Moortele PF, Akgun C, Adriany G, Moeller S, Ritter J, Collins CM, Smith MB, Vaughan JT, Ugurbil K. B1 destructive interferences and spatial phase patterns at $7 \mathrm{~T}$ with a head transceiver array coil. Magn Reson Med 2005;54:1503-1518.

[15] Kelley DAC. Characterizing in vivo B1 Maps at 7T using the Kolmogorov-Smirnov Test. In Proceedings of the $23^{\text {rd }}$ Annual Meeting of ISMRM, Toronto, Canada, 2015. p. 2385.

[16] Graesslin I, Homann H, Biederer S, B“ornert P, Nehrke K, Vernickel P, Mens G, Harvey P, Katscher U. A specific absorption rate prediction concept for parallel transmission MR. MR. Magn Reson Med 2012;68:1664-1674.

[17] de Greef M, Ipek O, Raaijmakers AJE, Crezee J, van den Berg CAT. Specific absorption rate intersubject variability in 7T parallel transmit MRI of the head. Magn Reson Med 2013;69:1476-1485.

[18] Nistler J, Diehl J, Renz W, Eberler L. Homogeneity improvement using a 2 port birdcage coil. In Proceedings of the 15th Annual Meeting of ISMRM, Berlin, Germany, 2007. p. 1063.

[19] Schmitter S, Wu X, Ugurbil K, Van de Moortele P-F. Design of parallel transmission radiofrequency pulses robust against respiration in cardiac MRI at 7 Tesla. Magn Reson Med 2015;74:1291-1305.

[20] Mugler JP, Brookeman JR. Three-dimensional magnetization-prepared rapid gradientecho imaging (3D MP RAGE). Magn Reson Med 1990;15:152-157.

[21] Setsompop K, Wald LL, Alagappan V, Gagoski B, Adalsteinsson E. Magnitude least squares optimization for parallel radio frequency excitation design demonstrated at 7 Tesla with eight channels. Magn Reson Med 2008;59:908-915. 
[22] Medical electrical equipment-part 2-33: particular requirements for the basic safety and essential performance of magnetic resonance equipment for medical diagnosis. 3rd ed Geneva: International Electrotechnical Commission, 2010. 603:2-33.

[23] Hoyos-Idrobo A, Weiss P, Massire A, Amadon A, Boulant N. On Variant Strategies to Solve the Magnitude Least Squares Optimization Problem in Parallel Transmission Pulse Design and Under Strict SAR and Power Constraints. IEEE Trans on Med Imag 2014;33:739748.

[24] Guérin B, Gebhardt M, Cauley S, Adalsteinsson E, Wald LL. Local specific absorption rate (SAR), global SAR, transmitter power, and excitation accuracy trade-offs in low flip-angle parallel transmit pulse design. Magn Reson Med 2014;71:1446-1457.

[25] Eichfelder G, Gebhardt M. Local specific absorption rate control for parallel transmis- sion by virtual observation points. Magn Reson Med 2011;66:1468-1476.

[26] Lee J, Gebhardt M, Wald LL, Adalsteinsson E. Local SAR in parallel transmission pulse design. Magn Reson Med 2012;67:1566-1578.

[27] Massire A, Cloos MA, Luong M, Amadon A, Vignaud A, Wiggins CJ, Boulant N. Thermal simulations in the human head for high field MRI using parallel transmission. J Magn Reson Imag 2012;35:1312-1321.

[28] Ferrand G, Luong M, Amadon A, Boulant N. Mathematical tools to define SAR margins for phased array coil in-vivo applications given E-field uncertainties. In Proceedings of the 23rd Annual Meeting of ISMRM, Toronto, Canada, 2015. p. 3763.

[29] Gumbrecht R. Development of customized pTx MR excitation methods and their safe application. Friedrich-Alexander-Universität Erlangen-Nürnberg, Erlangen 2013. Ph.D thesis. https://opus4.kobv.de/opus4-fau/files/3860/Doktorarbeit.pdf.

[30] Amadon A, Mauconduit F, Vignaud A, Boulant N. Slice profile corrections in the XFL (Magnetization Prepared Turbo Flash) B1 mapping sequence. In Proceedings of the 23rd Annual Meeting of ISMRM, Toronto, Canada, 2015. p. 2377.

[31] Fautz HP, Vogel M, Gross P, Kerr A, Zhu Y. B1 mapping of coil arrays for parallel transmission. In Proceedings of the 16th Annual Meeting of ISMRM, Toronto, Canada, 2008. p. 1247. 
[32] Brunner DO, Pruessmann KP. B1+ interferometry for the calibration of RF transmitter arrays. Magn Reson Med 2009;61:1480-1488.

[33] Smith SM. Fast robust automated brain extraction. Human Brain Mapping 2002;17:143155.

[34] Gras V, Luong M, Amadon A, Boulant N. Joint design of $\mathrm{k}_{\mathrm{T}}$-points trajectories and RF pulses under explicit SAR and power constraints in the large flip angle regime. J Magn Reson 2015;261:181-189.

[35] Silver MS, Joseph RI, Hoult DI. Selective spin inversion in nuclear magnetic resonance and coherent optics through an exact solution of the Bloch-Riccati equation. Phys Rev A 1985;31: 2753-2755.

[36] Ashburner J, Friston KJ. Unified segmentation. NeuroImage 2005;26:839-851.

[37] Boulant N, Le Bihan D, Amadon A. Strongly modulating pulses for counteracting RF inhomogeneity at High fields. Magn Reson in Med 2008;60:701-708.

[38] Amadon A, Dupas L, Vignaud A, Boulant N. Does the best distance between 2 spokes match the inverse RF wavelength? In Proceedings of the 23rd Annual Meeting of ISMRM, Toronto, Canada, 2015. p. 2388.

[39] Cloos MA, Wiggins C, Wiggins G, Sodickson D. Plug and play parallel transmission at 7 and 9.4 Tesla based on principles from MR fingerprinting. In Proceedings of the 22nd Annual Meeting of ISMRM, Milan, Italy, 2014. p. 542.

[40] Boulant N, Wu X, Adriany G, Schmitter S, Ugurbil K, Van de Moortele P-F. Direct control of the temperature rise in parallel transmission by means of virtual observation points: simulations at 10.5 T. Magn Reson Med 2015. doi: 10.1002/mrm.25637.

[41] Moore J, Grissom WA, Gore JC. Fast Prediction of RF fields in the human brain. In Proceedings of the 23rd Annual Meeting of ISMRM, Salt Lake City, Utah, USA, 2013. p. 2593. 
a)

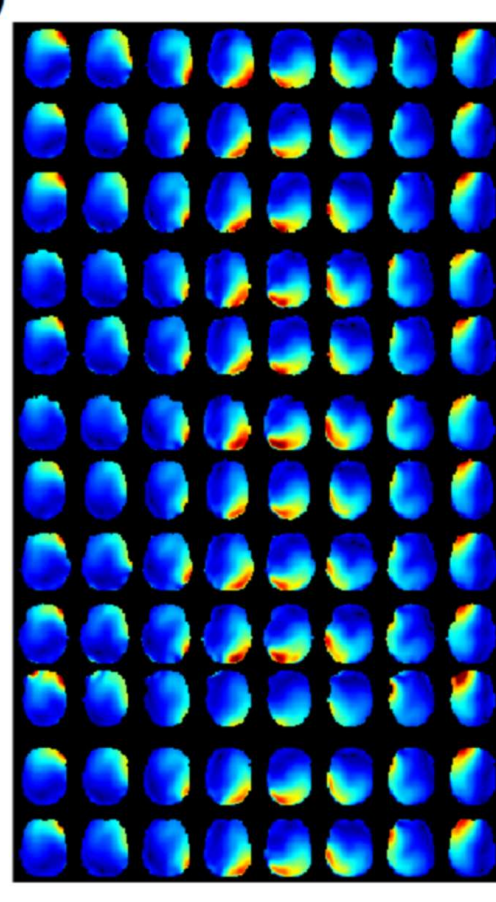

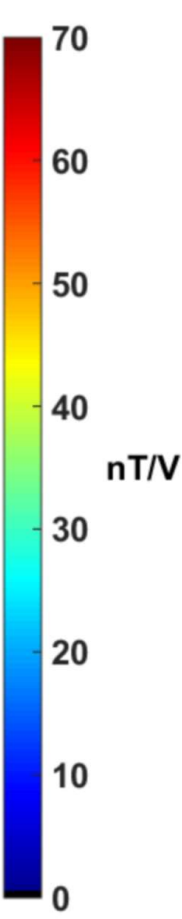

b)

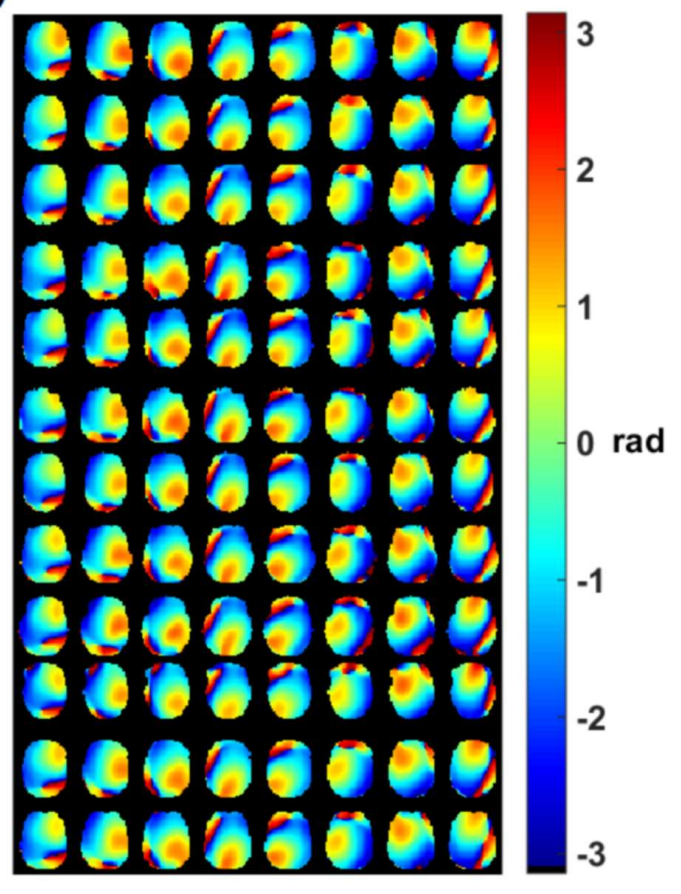

Fig. 1. Measured B1 maps (axial view) in a) amplitude and b) phase at isocenter. In each subplot, images are sorted according to subject (\#1-12 from top to bottom) and channel indexes (\#1-8 from left to right). The phase shown in b) is relative with respect to a common CP-mode. 


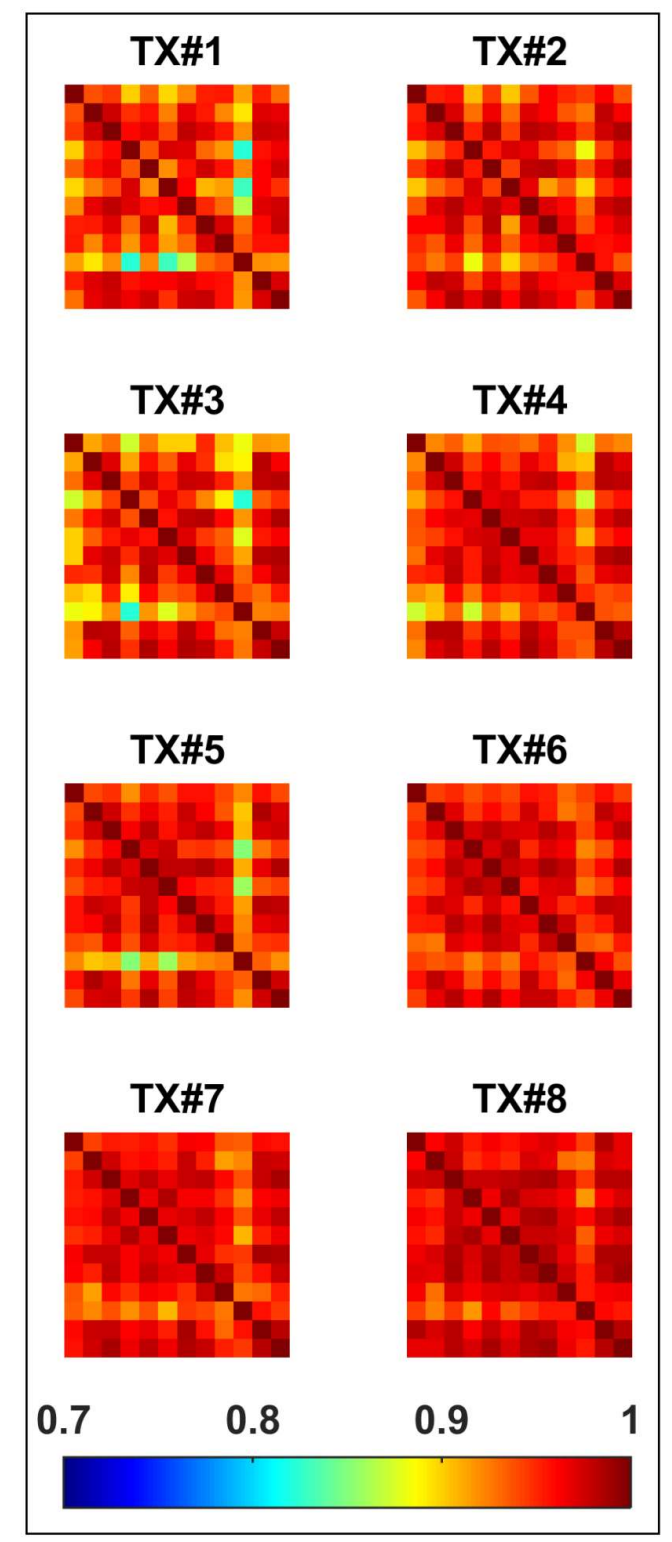

Fig. 2. Channel-by-channel (TX\#1-8) correlations between the B1 maps measured over 12 subjects. The worst-case correlation is 0.82 and occurs between subjects \#4 and \#10 with channel TX\#1. The averages ( \pm standard deviation) for the 8 channels are respectively: $0.94 \pm$ $0.03,0.95 \pm 0.02,0.94 \pm 0.03,0.95 \pm 0.03,0.95 \pm 0.03,0.96 \pm 0.02,0.96 \pm 0.02,0.97 \pm 0.02$. The calculation takes into account the phase of the field maps. 
a)

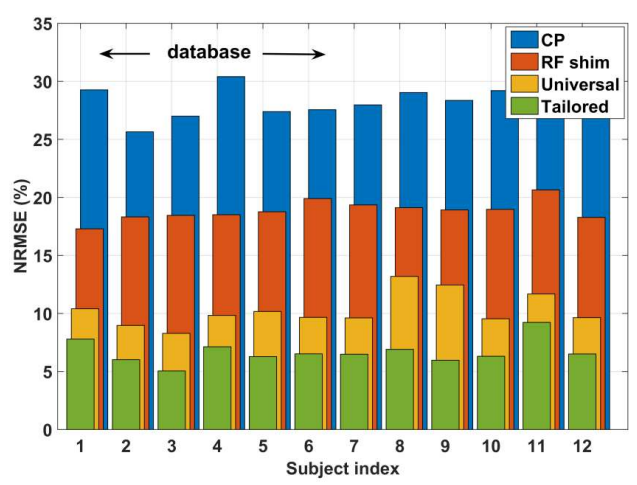

c)

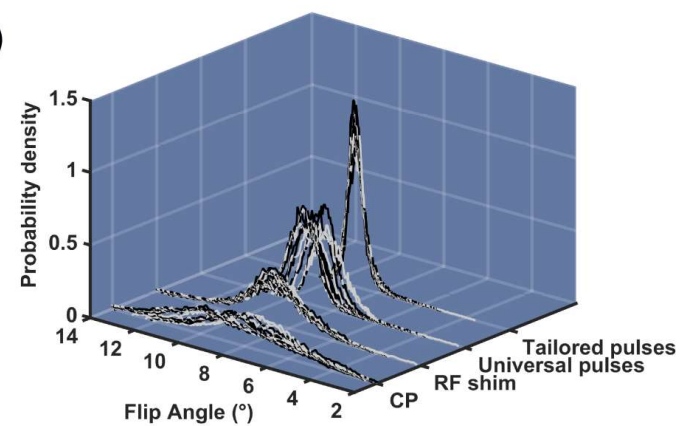

b)

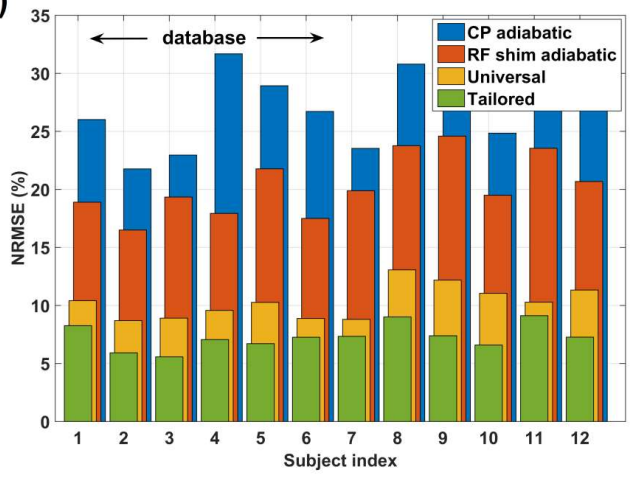

d)

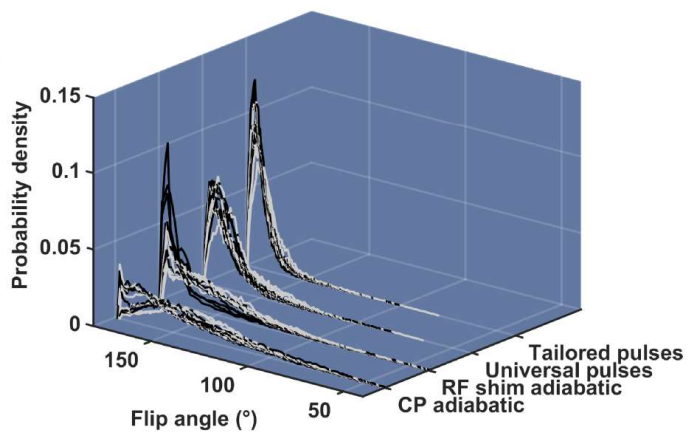

Fig. 3. a-b) NRMSEs respectively for the excitation and inversion pulses across all subjects (blue: CP, orange: RF shim, yellow: universal pulses, green: tailored), and c-d) corresponding FA histograms for the different pulse techniques and for the different subjects. In $a-b)$ subjects \#1-6 constitute the database over which the design of the universal pulses was performed. In cd) the black and gray lines correspond to the FA histograms of the subjects contained and not contained in the database respectively. 


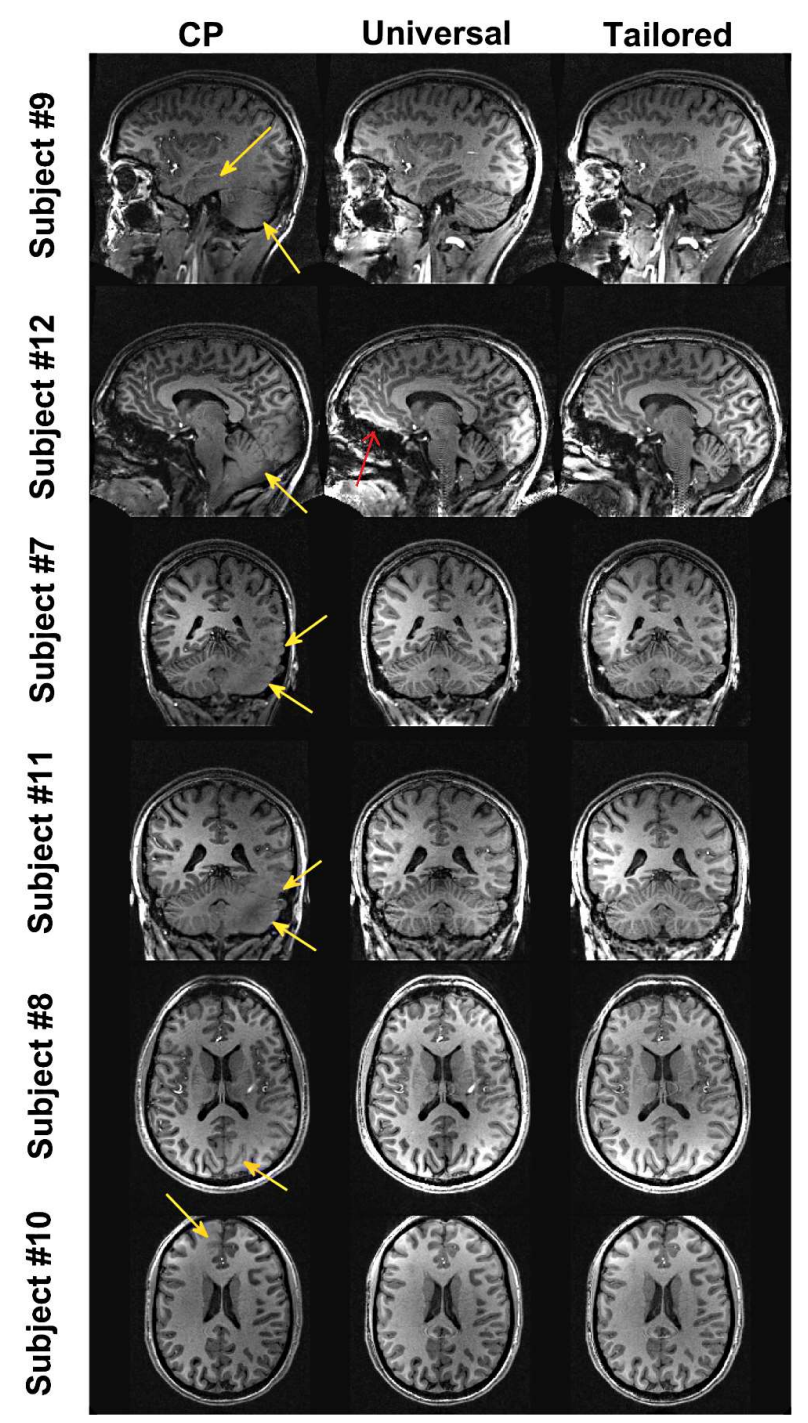

Fig. 4. Native (no reception profile correction) 7T MPRAGE images for subjects \#7-12. From left to right: CP-mode, universal and subject-based tailored pulses. The universal pulses are able to greatly mitigate the RF field inhomogeneity problem without $\mathrm{B} 1$ and $\Delta \mathrm{B} 0$ measurements (yellow arrows), while satisfying various hardware and safety constraints. The red arrow points to a small susceptibility artefact which is better compensated by the subject-based tailored pulses. Some artefacts are also visible in the brainstem of subject \#12 and are due to the nonlinearity of the used head gradient system (shoulder artefact). 


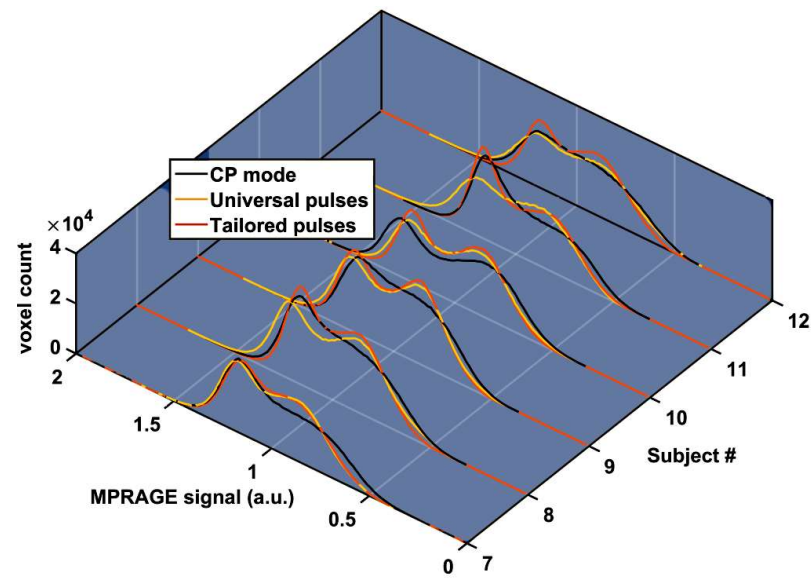

Fig. 5. Histograms of the MPRAGE signal intensity over the pooled white and gray matter masks for subjects \#7-12 for the CP-mode (black), universal (yellow) and subject-specific tailored (red) RF pulses. The signal intensity was analyzed after bias field correction and segmentation, performed by SPM12. The gray matter pool, corresponding in the histogram to the hump with the lowest signal, is more pronounced when switching from the CP mode to the universal pulses.
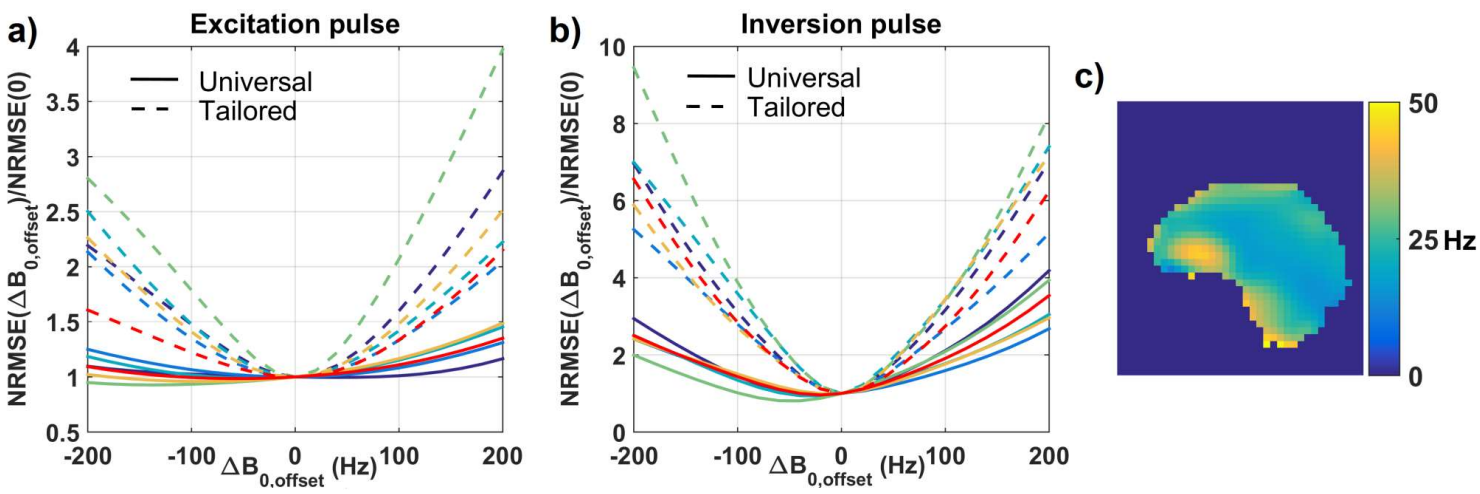

Fig. 6. Normalized deviation of the NRMSE versus additional $\Delta \mathrm{B} 0$ offset for the a) excitation and $\mathrm{b}$ ) inversion pulses. In the Bloch simulations, this $\Delta \mathrm{B} 0$ offset was added as a global offset to the measured $\Delta \mathrm{B} 0$ maps. The solid lines correspond to the universal pulses (designed using subjects \#1-6) while the dashed lines correspond to the subject-based tailored pulses. Each color corresponds to a different subject (subjects \#7-12). The subject-to-subject variation of $\Delta \mathrm{B} 0$ is shown in c) with the map of the standard deviation calculated over the different subjects and represented over the intersection of their respective masks. 
